\title{
Short stay facilities: the future of efficient paediatric emergency services
}

\author{
G J Browne, A Penna
}

\begin{abstract}
Many children admitted to hospital can stay for 24 hours or less. Short stay facilities offer such children rapid stabilisation and early discharge with considerable financial saving. A 12 month study was completed in which data were collected from the children's emergency annex (CEA) at Westmead Hospital in Sydney's western suburbs. This university based teaching hospital provides care for a large paediatric population as well as three other district hospitals with limited children's bed capacity. From April 1994 to April 1995, 1300 children were admitted and entered into a database of general and hospital-specific information. Critical incident monitoring was undertaken and follow up with review within 24-72 hours for all children. The CEA increased hospital efficiency significantly by reducing bed days, with a saving of over $\$ 500000$ to the department. The average length of stay was 17.5 hours, and $58 \%$ of users were children of 2 years and under. Only 3\% remained beyond 24 hours, and another $4 \%$ were admitted to inpatient beds for continued management of the primarily diagnosed condition. No critical incident was reported during this 12 month period. Short stay facilities are efficient and cost-effective for children with acute illness who can be rapidly stabilised with early discharge without critical incident. Children 12 months and under are particularly suited to this type of facility. Short stay facilities should be used to augment efficiency within children's emergency services which have high turnover and limited bed capacity. (Arch Dis Child 1996; 74: 309-313)
\end{abstract}

Keywords: illness, emergency, short stay.

Children's Emergency

Department of Paediatric Critical Care, Westmead Hospital, Sydney, Australia

G J Browne

Department of Paediatrics, Westmead Hospital, Sydney, Australia

A Penna

Correspondence to: G J Browne, Emergency Department, New Children's Hospital, Hawkesbury Road, Westmead, 2145 Sydney, Australia.

Accepted 4 January 1996
Health services are under great pressure to be efficient in medical care and in cost to the community. Children's emergency departments have added pressures of parental anxiety and high expectations for rapid and efficient service. Acute medical services may find it difficult to comply at all times with new directives. This may lead to overspending and the need to recoup costs from other areas.

Many children are admitted to a paediatric ward for observation either because illness is evolving or when it is uncertain how the child will respond. Continuity of care is easily lost when assessment is handed over to medical staff unfamiliar with the case. This can lead to delay in medical review and may interrupt important treatment ordered in the emergency department. Many children with acute illness stabilise rapidly and can be discharged within 24 hours. The present emergency system does not allow for their extended observation or early discharge. Short stay facilities should allow a more efficient and flexible approach.

We created a database, updated daily, to examine the outcome of children admitted to a short stay facility over a 12 month period. So far as we know these data are the first to assess the functioning of a fully serviced children's short stay facility.

\section{Background}

A recent inpatient study showed that many children required admission for less than 24 hours, but discharge was delayed due to delay in medical review. 1 A retrospective review of the case records of 4000 admissions to Westmead Hospital during 1992 showed that 1000 children (25\%) could have stayed less than 24 hours, as the admitting problem had stabilised rapidly allowing discharge home; $750(75 \%)$ of these, although stable, remained in hospital unnecessarily waiting for team review before discharge home. These children had prolonged admission which could have been avoided (Browne GJ, Penna A, unpublished data). This was the driving force for the development of a short stay facility, the children's emergency annex (CEA).

Westmead Hospital is a university based teaching hospital, servicing the north-western area of Sydney, which has a population of 1.7 million. This region has a large ethnic mix, and three district centres have small general children's units that use Westmead Hospital as their primary referral centre. These hospitals service over 350000 children. Westmead Hospital also serves the primary needs of its immediate region, accounting for one third of the population. The hospital also provides intensive care facilities and a major trauma service for the north-west region. The capacity of the children's wards is limited to 68 beds, with four intensive care and six high dependency beds. Regional policy is for transfer of acutely or seriously ill children to Westmead Hospital. Tertiary referrals, however, are sometimes made to The Royal Alexandra Hospital for Children, a university based centre out of region, at a considerable distance.

The emergency services for children include dedicated resuscitation and trauma beds, observation beds, cubicles, procedure and minor resuscitation area, and the CEA, established in March 1994. The CEA was 
Table 1 Patient categories appropriate for admission to the children's emergency annex

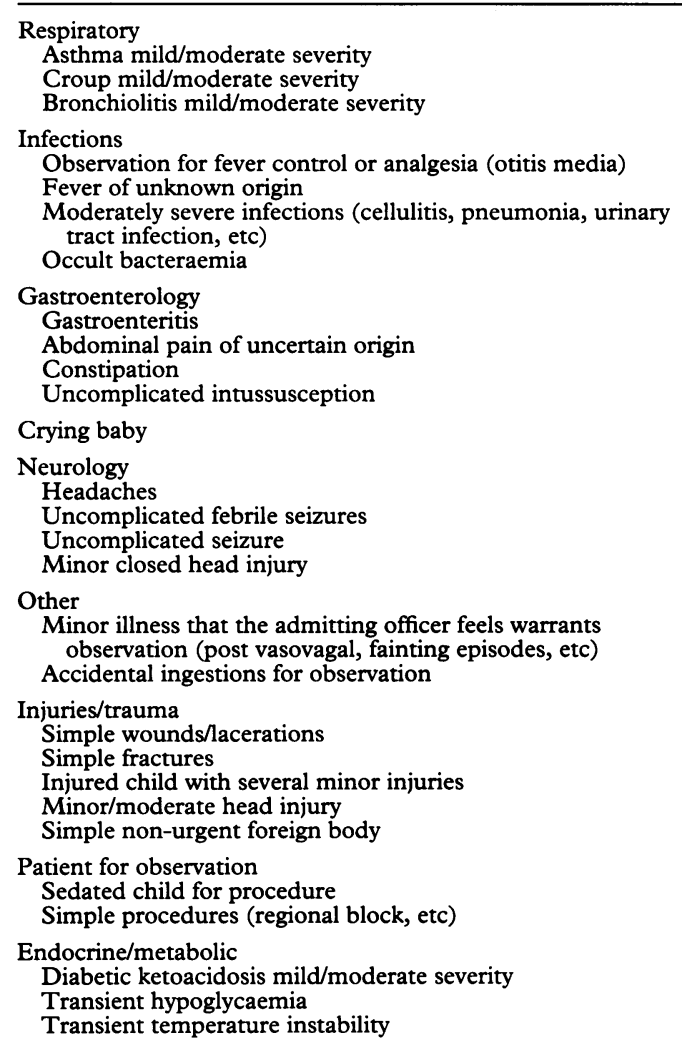

developed to accommodate a minimum of four short stay beds with all necessary equipment, located within the children's emergency area and fully equipped for resuscitation and stabilisation, with a strict budget of $\$ 20000$. Equipment had to be multipurpose, and physical changes within the department were only those essential for efficient running of the service. Policies and procedures were developed and implemented to suit the needs of those children coming to the emergency department. Other issues considered were children with infectious diseases needing an isolation room and greater involvement of nursing and medical staff in decision making.

The acute care team was introduced to oversee this new role. The team consisted of an acute care consultant, a medical registrar, and nursing staff available 24 hours a day.

\section{Methods}

PATIENT POPULATION

All children presenting to the emergency department of Westmead Hospital were considered for possible admission to the CEA.

Table 2 Patient categories inappropriate for admission to the children's emergency annex

Require more than 24 hours of treatment or nursing care May need intensive care or high dependency facilities Can be discharged within 4 hours

Require more than basic nursing care

Require more than basic nursing
Require complicated treatments

Require complicated treatments
Require routine admission to the main ward area

Require routine admission to the main
Require postoperative monitoring

Require continuous monitoring (except pulse oximetry, blood pressure)
presuire cont
Over the 12 months from April 1994 to April 1995, 13150 children presented to the emergency department for assessment, and 4208 $(32 \%)$ were admitted to inpatient beds or the CEA. During this period 1300 children were admitted to the CEA, accounting for $30 \%$ of the total admissions for children to Westmead Hospital.

\section{DATA COLLECTION}

The 1300 children admitted from April 94 to April 95 were entered into a database by the admitting nurse and doctor. This included demographic information, referral address, home address, and discharge destination. General patient information was also recorded with both initial and final diagnosis, age of the child, and length of the child's stay in the CEA. The appropriateness of the child's stay was determined from protocols (tables 1 and 2) and was the decision of the acute care consultant of the day.

Follow up was organised and the information obtained recorded for all children discharged from the CEA. Contact with the on call acute care consultant was encouraged should a child deteriorate before follow up could occur. Parents and follow up doctors were asked to inform the department if follow up did not occur, so this could be recorded. Critical incident monitoring reports were completed by the nurse, doctor, or parents for all adverse events within three days of discharge. The critical incident reports were those developed by the Australasian College for Emergency Medicine, August 1993.2

\section{OUTCOME MEASURES}

Outcome measures for the operation of the CEA were based upon appropriateness of stay, time of discharge, adverse events during admission, readmission after discharge, number of admissions to hospital from the CEA, and adverse events reported within three days of discharge. Appropriateness of stay was determined by the acute care consultant according to the working policy of the CEA, and documented in the database by the acute care consultant.

In addition, cost savings were calculated by the difference in cost between an inpatient bed and a bed in the CEA. This was determined independently by our hospital administration at $\$ 250$.

\section{DATA ANALYSIS}

The data were analysed to determine the common diagnoses, age distribution of use, efficiency of throughput, and outcome of children admitted to the CEA. The overall benefit of the unit in cost saving was undertaken using bed days as an end point. Bed days over the preceding four years (1990 to 1993) were compared with bed days over the period of our 12 month study. These data were then compared using the Student $t$ test. 


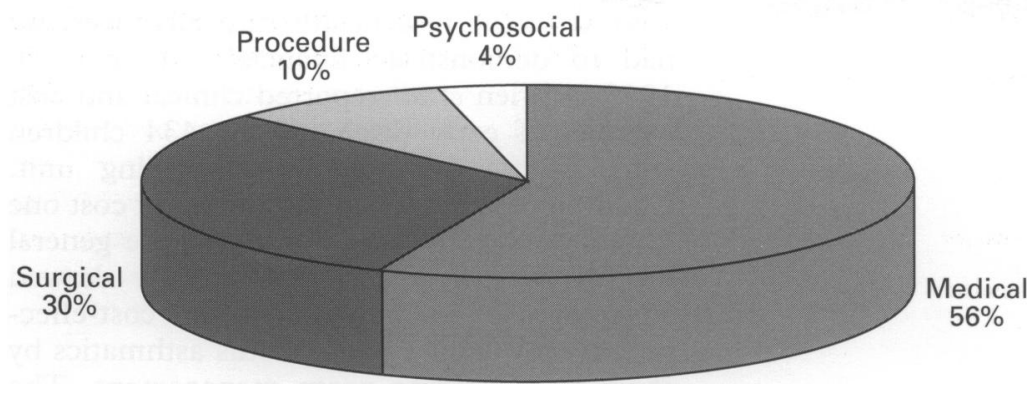

Figure 1 Breakdown of types of admission.

\section{Results}

Of 13150 acute presentations to the emergency department over the 12 month period of the study, 1300 children were admitted to the CEA. The remaining 2892 admissions were deemed sick enough or satisfied criteria for direct admission to the main children's ward, with $273(10 \%)$ of these children admitted to intensive care/high dependency.

Patients admitted to the CEA are shown in fig 1. Medical cases accounted for 728 admissions (56\%). They consisted mainly of respiratory illness such as asthma, croup, bronchiolitis, and pneumonia, gastroenteritis, seizures, ingestions, environmental problems such as minor near drowning episodes, risk of envenomation by spider, heat exhaustion, infections such as occult bacteraemia and cellulitis, and mild diabetic ketoacidosis. Surgical cases accounted for 390 admissions (30\%). The most common diagnoses were trauma (most being head injury, fractures, lacerations, simple wound care) and abdominal pain. Procedures or postprocedure recovery accounted for 130 admissions (10\%). These included recovery from intravenous regional anaesthesia, postoperative observation, and children having intravenous rehydration, antibiotics, or blood tests. Also included were children recovering from procedures such as air reduction of intussusception. Psychosocial problems accounted for 52 admissions (4\%). The commonest diagnoses included intentional overdose, adolescent crisis, behavioural reactions, family stress, and observation of crying infants (table 3 ).

The main users of the CEA were children under 2 years, accounting for $58 \%$ of all

Table 3 Most common admissions to the children's emergency annex

\begin{tabular}{ll}
\hline Medical ( $n=728 ; 56 \%)$ & Surgical $(n=390 ; 30 \%)$ \\
Respiratory (291) & Trauma (172) \\
Asthma & Head injury \\
Croup & Blunt abdominal injury \\
Bronchiolitis & Lacerations \\
Pneumonia & Facial/oral injury \\
Gastroenteritis (150) & Dental injury \\
Convulsion (110) & Simple fracture (117) \\
Febrile/afebrile & Upper limb (90\%) \\
Fever for observation (75) & Lower limb (10\%) \\
Occult bacteraemia (40) & Abdominal pain (47) \\
Viral illness/fever control (20) & Wound infection/debridement (31) \\
Environmental problems (20) & Post-treatment (23) \\
Near drowning & Air reduction intussusception \\
Spider bite & Foreign body removal \\
Heat exhaustion & \\
Accidental ingestion (18) & \\
Diabetic keroacidosis (4) & \\
Emergency procedure $(n=130 ; 10 \%)$ & Psychosocial $(n=52 ; 4 \%)$ \\
Intravenous rehydration (91) & Crying/irritable infant (37) \\
Antibiotic administration (20) & Behavioural disorder (6) \\
Bier's block (12) & Adolescent crisis (5) \\
Blood collection (7) & Overdose (4) \\
\hline
\end{tabular}

admissions. Almost two thirds of these were less than 12 months of age. The remainder were aged up to 15 years (fig 2). Nine hundred and ten cases $(70 \%)$ were admitted between 1200 and 2000 hours, with $260(20 \%)$ admitted between 2000 and 0100 hours. The main peak of activity in the short stay annex is after normal working hours. Only 65 patients $(5 \%)$ were admitted after 0100 hours, with a further 65 admissions (5\%) occurring between 0900 and 1200 hours.

The average length of stay was 17.5 hours, $39(3 \%)$ stayed over 24 hours in the department (fig 3), with $26(2 \%)$ of them being the result of delays in inpatient bed availability. This occurred predominantly during two periods when there were epidemics of bronchiolitis and gastroenteritis.

Only 13 admissions (1\%) were deemed inappropriate (table 2), although 52 children $(4 \%)$ were transferred to the ward because their condition required inpatient care. No critical incidents were reported in the CEA over the 12 month study period. Eighty five per cent of children were followed up within three days by either the local medical officer or a paediatrician. No adverse discharge events were documented in children discharged from the CEA. The four children who returned for readmission had minor problems but high parental anxiety.

The CEA significantly reduced bed days. The mean yearly admissions for 1990 to 1993 were 5315. Admissions in 1994 were 4766 . This reduction in bed days is statistically significant, as determined by the Student $t$ test $(p=0.0072)$. Bed days at a differential bed cost (hospital bed $v$ CEA bed) of $\$ 250$ a day saved up to $\$ 500000$.

\section{Discussion}

No study has shown whether a broad range of acute illness in children can be accommodated efficiently and safely in a short stay facility with successful cost savings. The CEA allowed for longer observation periods in the emergency setting, particularly for small infants in whom non-specific symptoms and signs can evolve into serious illness, and for stabilisation of these children and continuity of care in a safe environment. This continuity of care allowed for a broader range of disposition options for physicians, as more certain diagnoses could be made. It also removed uncertainty of early discharge for those children with labile or unpredictable responses to an acute illness.

The development of our children's emergency annex occurred after much planning and concentrated effort, with the focus on four areas: the physical layout of the CEA, the development of a budget, the development of organisational and operational policies, and the transfer of control over admission and discharge from the paediatrician or surgeon to the acute care consultant.

Strict admission criteria excluded critically ill children who needed hospital or high dependency care. The acute care team undertook rounds twice daily and assessed children at 


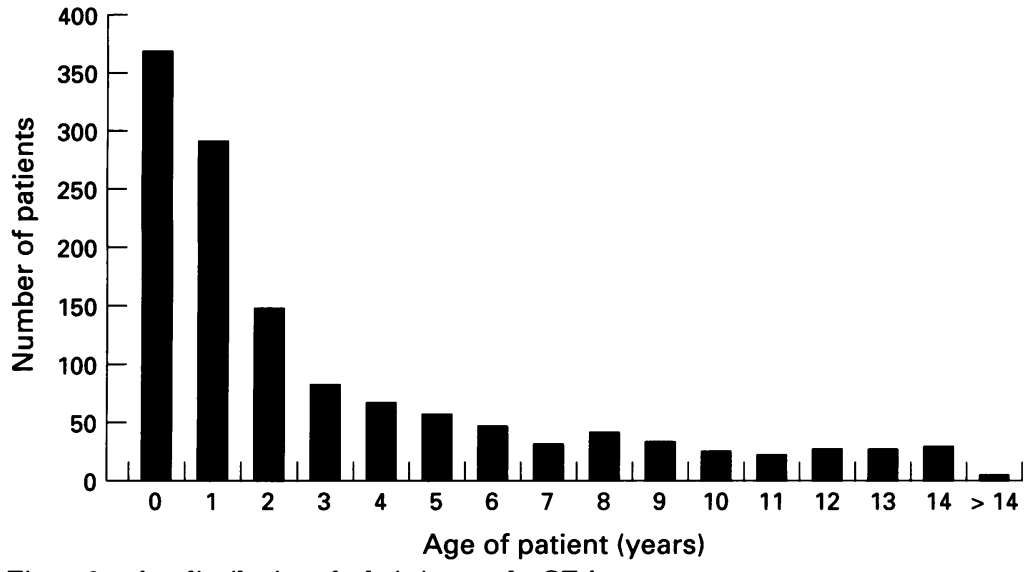

Figure 2 Age distribution of admissions to the CEA.

regular intervals throughout the day and night to avoid the CEA becoming a holding ward for inpatients. The acute care consultant assessed all cases and if admission to the ward became necessary it was organised by the paediatric admitting officer.

Admission criteria have to be broad enough to encompass a wide range of emergency conditions requiring more than four hours of care, and yet allow for safe discharge within 24 hours. The large number of medical cases admitted (56\%) reflects the typical work load of a busy children's emergency service. It also highlights the ability to stabilise rapidly many acute medical illnesses in children, especially croup, asthma, and bronchiolitis, as observed by O'Brien et al in their study on children with asthma. ${ }^{3}$ Surgical cases admitted to our unit (table 3) had similar problems to those in other units, ${ }^{4}$ with trauma, including fractures, dominating. Head injury accounted for $50 \%$ of admitted cases. ${ }^{5}$ Because we are a major trauma centre we allowed a broader range of trauma admissions, such as children with moderate blunt abdominal injuries stable after initial investigation and observation and likely to be discharged within 24 hours.

The flexible nature of the CEA proved useful in the successful modification of the management of certain targeted conditions such as diabetic ketoacidosis.

Acute behavioural issues were better managed and observed in the CEA. Many parents and children with such problems are very anxious and this anxiety may be increased through ward admission, giving the parent the impression their child is truly seriously ill

For the CEA to be successful within the

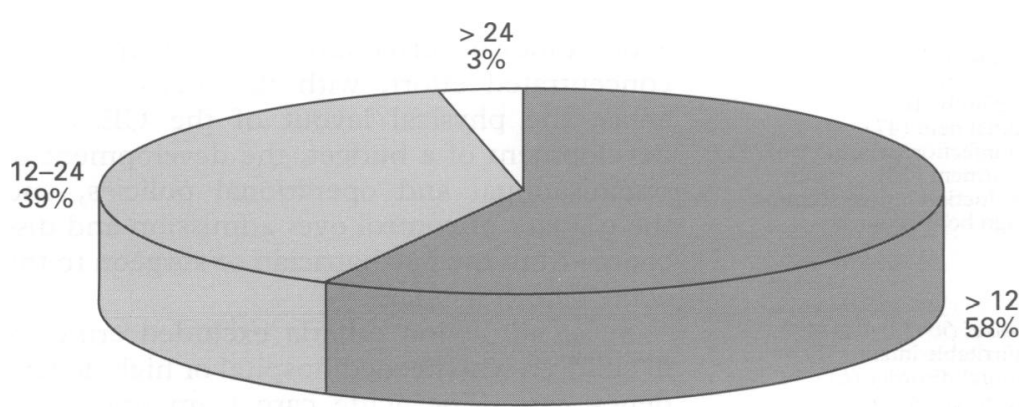

Figure 3 Duration of admission to the CEA (hours). context of the new health care directives, we had to demonstrate financial efficiency. In 1980 O'Brien $e t a l^{\beta}$ reported clinical and cost benefits of early discharge for 434 children with asthma admitted to a holding unit. Children cared for in the holding unit cost one fifth that of children admitted to the general hospital wards. Willert et $a l^{6}$ clearly showed advantages of early discharge and cost-effectiveness for children with status asthmatics by short term holding room management. The CEA has demonstrated significant savings with reduction in bed days $(p=0.0072)$ and an estimated $\$ 500000$ in savings.

Beattie and Moir $^{4}$ showed, through their experience of a small short stay facility at the Royal Aberdeen Children's Hospital, that efficient and safe management of children can occur; however, they limited their admissions mostly to surgical trauma cases and excluded all children under 12 months of age. The development of this age criterion may have been related to studies suggesting that head injury in older children can be managed with observation and early discharge. ${ }^{5} \mathrm{~A}$ feature of the CEA which stands out in comparison to this study is the large number of children under 2 years of age. We have found this group difficult to assess acutely in an emergency department setting without continuing observation. Almost $95 \%$ of children aged 12 months or less were followed up within 24 to 48 hours. This is an important factor in their care.

The average length of stay was 17.5 hours, with only a few staying beyond 24 hours. This is consistent with other studies ${ }^{4}$ and allowed adequate stabilisation of children with a sufficient period of observation to decide upon discharge home.

Transfers into inpatient beds occurred in only $4 \%$, as in other studies. ${ }^{4}$ The most common were children with asthma, bronchiolitis, and vomiting from gastroenteritis, and infants with evolving sepsis.

The CEA requires support from social work and adolescent services, community and family services, nurse educators, and ancillary services such as physiotherapy and dietetics.

We believe parental involvement increased, with many feeling an integral part of their child's management. We considered parents were more willing to highlight any underlying concern they had about their child's illness. The opportunity to observe families closely made it possible to be more objective over their ability to cope at home. Many require only support and encouragement but a small minority required more input from ancillary services and more formalised follow up before discharge.

We also found that a stay in the CEA provided a window of opportunity to view community needs of children. We are concerned at the increasing number of adolescents being admitted to the CEA with intentional overdose or adolescent crisis and intend a further review of their management.

The CEA increases bed efficiency for inpatients and emergency patients and has given us flexibility to cope during periods when 
workload is high. It manages sick children efficiently, maintaining a high turnover with safety. It improves bed utilisation, with more parental involvement and considerable financial savings. It helps us to modify and develop new medical practices in a safe environment for example, the management of mild diabetic ketoacidosis - out of the main hospital. It has become an important link between the community and the hospital, with high consumer satisfaction.

We would like to thank the paediatricians of Westmead Hospital and Dr P Gaudry for their enthusiasm and continued support of the CEA. We would also like to thank all of the nursing staff and junior doctors who gave so much energy and time into making the CEA function efficiently. A special dedication to the late Dr Reuben Dubois whose foresight and vision created the concept of the children's emergency annex.

1 Numa A, Oberklaide F. Can short hospital admissions be avoided ? Med $\mathcal{F}$ A ust 1991; 155: 395-8.

2 Vinen JD, Gaudry PL, Ashby $\mathrm{R}$, et al. Critical incident monitoring study (CIMS) in emergency medicine: an interim monitoring study (CIMS) in emertim Human Services and Health, 1994.

3 O'Brien AR, Heinet EW, Sly MR. Treatment of acute asthmatic attacks in a holding unit of a paediatric emerasthmatic attacks in a holding unit of a paed

4 Beattie TF, Moir PA. Paediatric accident and emergency short-stay ward: a 1 year audit. Arch Emerg Med 1993; 10: 181-6.

5 Sainsbury CPO, Sibert JR. How long do we need to observe head injuries in hospital ? Arch Dis Child 1984; 59: 856-9. 6 Willert C, Davis AT, Herman J, et al. Short-term holding room treatment of asthmatic children. $\mathcal{F}$ Pediatr 1985; 106: 797-11.

\section{Rotavirus immunisation}

Should children be immunised against rotavirus? Attenuated live oral vaccines are being developed and preliminary trials have suggested that they would be 50 to $80 \%$ effective in preventing rotavirus diarrhoea. It is anticipated that rotavirus vaccines might be licensed for use in the USA by 1996 . They would be given alongside diphtheria, pertussis, and tetanus, oral polio, and Haemophilus influenzae B immunisation.

Whether a vaccination programme is worthwhile depends not only on the efficacy and cost of the vaccine but also on the prevalence and severity of the disease. In the US rotavirus infection is almost universal in the first five years of life; it causes over three million cases of diarrhoea, between 65 and 70 thousand hospital admissions, and 125 deaths of infants and young children each year. A cost effectiveness analysis (Jean C Smith and colleagues, Pediatrics 1995; 96: 609-15) has come out in favour of universal immunisation.

Taking the lowest anticipated efficacy of $50 \%$ it has been calculated that universal vaccination would save the American health care system some $\$ 78$ and the society at large $\$ 459$ per case prevented. The vaccination programme would cost $\$ 243$ million a year but would save $\$ 79$ million in health care costs and $\$ 466$ million in terms of work productivity and non-medical costs. It is estimated that vaccination would prevent at least a million cases of rotavirus diarrhoea, 58000 hospitalisations, and 82 deaths each year.

These authors conclude that a rotavirus immunisation programme would be cost effective in the US. Presumably other countries will need to perform a similar assessment.

ARCHIVIST 